\title{
Changes in muscle strength and pain in response to surgical repair of posterior abdominal wall disruption followed by rehabilitation
}

\section{A E Hemingway, L Herrington, A L Blower}

See end of article for authors' affiliations

\section{Correspondence to:}

Miss Hemingway, Cheshire Physiotherapy Centre, 68a Barrington Road, Altrincham, Cheshire, UK andreahemingway@ hotmail.com

Accepted 29 April 2002

\begin{abstract}
Background: Posterior abdominal wall deficiency (PAWD) is a tear in the external oblique aponeurosis or the conjoint tendon causing a posterior wall defect at the medial end of the inguinal canal. It is often known as sportsman's hernia and is believed to be caused by repetitive stress.

Objective: To assess lower limb and abdominal muscle strength of patients with PAWD before intervention compared with matched controls; to evaluate any changes following surgical repair and rehabilitation.

Methods: Sixteen subjects were assessed using a questionnaire, isokinetic testing of the lower limb strength, and pressure biofeedback testing of the abdominals. After surgery and a six week rehabilitation programme, the subjects were re-evaluated. A control group were assessed using the same procedure.

Results: Quadriceps and hamstrings strength was not affected by this condition. A deficit hip muscle strength was found on the affected limb before surgery, which was significant for the hip flexors $(p=$ 0.05). Before surgery, $87 \%$ of the patients compared with $20 \%$ of the controls failed the abdominal obliques test. Both the injured and non-injured sides had improved significantly in strength after surgery and rehabilitation. The strength of the abdominal obliques showed the most significant improvement over the course of the rehabilitation programme.

Conclusions: Lower limb muscle strength may have been reduced as the result of disuse atrophy or pain inhibition. Abdominal oblique strength was deficient in the injured patients and this compromises rotational control of the pelvis. More sensitive investigations (such as electromyography) are needed to assess the link between abdominal oblique function and groin injury.
\end{abstract}

A bout $8 \%$ of all soccer injuries are to the groin. ${ }^{1}$ This value may be higher, but groin injuries do not always prevent a sportsman playing but only limit performance and are not recorded. Gilmore ${ }^{2}$ stated that the incidence of groin injury has increased because of increased intensity of play, but this is not supported by statistical evidence. ${ }^{1}$

Posterior abdominal wall deficiency (PAWD) was first recognised in the mid 1970s. ${ }^{3}$ It is believed that unusual or excessive stress such as caused by repeated kicking or rapid turning could result in a tear in the external oblique aponeurosis, the conjoint tendon, or a dehiscence between the inguinal ligament. The torn conjoint tendon causes a posterior wall defect at the medial end of the inguinal canal. ${ }^{45}$

The occurrence of this injury is not surprising considering that during kicking the hip has the highest net joint force and moment, with a ballistic impact, and these large forces are absorbed by the structures supporting the hip. ${ }^{6}$ After a kick, only $15 \%$ of the kinetic energy is absorbed by the ball ${ }^{7}$ the remaining force being dissipated throughout the body. The hip flexors forcefully contract during kicking to swing the leg forwards and downwards causing a repeated traction at the conjoint tendon. During sudden changes in direction while running, a forceful eccentric pull may also occur through the groin muscles. With repetition, these actions may cause fatigue and injury.

It has been suggested that there is an increase in the intraabdominal pressure during sport stressing the transversalis fascial fibres of the posterior inguinal wall. ${ }^{8}$ PAWD, also known as sportsman's hernia or Gilmore's groin, may also be caused by muscular imbalance. Strong and tight hip flexors tilt the pelvis forward and stretch the weak abdominal obliques. ${ }^{10}$ Janda and Schmid ${ }^{11}$ described this as pelvic crossed syndrome.
Eventually the muscle dysfunction causes tissue fatigue, and a tear in the abdominal obliques occurs. It has also been proposed that there is preferential recruitment of the rectus abdominis during running and turning, leaving the deep abdominals_-for example, the obliques — open to injury. ${ }^{4}$

The hip adductors and abductors are also thought to play an important role in pelvic stability. Hackney ${ }^{12}$ and Simonet et al $^{13}$ suggested that the adductor action produces a shearing force across the pubic symphysis causing excessive stress at the posterior inguinal wall. Lee ${ }^{14}$ described two muscular units that contribute to pelvic stability known as the inner and outer units. Two components of the outer unit are the anterior oblique and lateral system. The anterior oblique consists of the external and internal obliques and contralateral adductors. The lateral system consists of the opposite adductor to the abductor. These are thought to be important during ambulation, and, when weakness occurs, compensatory movements cause dysfunction to the pelvic region. It has been theorised that repetitive dysfunctional movements cause accumulative microtrauma and so injury. ${ }^{15}$ Research has found that a congenital posterior inguinal wall deficiency occurs in $25 \%$ of the male population. ${ }^{13} 16^{17}$ The theory of congenital weakness may be supported by the high percentage of bilateral disruptions found. Evans $^{8}$ found that $48 \%$ of male patients with PAWD had a bilateral disruption. Fevre ${ }^{18}$ argued that the high incidence in men was a result of the embryonic descent of the testis.

Despite all this information, there is a paucity of scientific research on how best to prevent and conservatively manage this condition. Surgery is the preferred mode of treatment followed by six to eight weeks of structured rehabilitation. ${ }^{5}$ Several studies have shown that groin pain and function are significantly improved after surgical repair. ${ }^{12} 131920$ Unfortunately most of 
these studies do not stand up to scientific scrutiny with poor rationale and methodology-for example, the sample size was small and standardised rehabilitation and surgical protocols were not used. None of these surgical studies have investigated the impact of a specific, progressive rehabilitation programme in conjunction with the surgery on strength, function, pain, and return to performance, despite the importance of intensive rehabilitation after surgery. ${ }^{10}$

Rehabilitation usually involves progressive resistance exercise for the lower limb and abdominals, a flexibility regimen, and a sports specific programme to attempt to restore the optimum function of the muscles. Stabilisation exercises aim to isolate appropriate muscles and retrain their holding capacity and coordination, alongside contraction with other synergists to support and protect the pelvic girdle. The hip flexors, abductors, and adductors originate at the pelvis, and more important than their mobilising role is their role in stabilising the contralateral side at the pelvis in activities such as kicking and turning. The rotation component of activities such as kicking and turning are thought to be a main cause of the microtears in the posterior inguinal wall. ${ }^{21}$ The abdominal obliques also have a stabilising role controlling rotational pelvic motion. The transverse abdominals have an important role in lumbar pelvic stability, and in normal subjects were found to be recruited first at the pelvis during movement. ${ }^{22}$ It was found that postural abdominal muscle endurance was reduced in subjects with lordotic postures and low back pain. ${ }^{23}$

This study aimed to assess lower limb and abdominal muscle strength, function, and pain level of patients with PAWD before surgery compared with matched controls, and then to compare these findings with the changes in muscle strength, pain, and function after surgery and six weeks of rehabilitation.

Therefore the null hypotheses for the study were:

(1) there will not be a deficit in the lower limb and abdominal muscle strength in patients with PAWD compared with matched controls;

(2) the rehabilitation protocol will not affect deficits in lower limb and abdominal muscle strength, pain, and function that occur following PAWD injury and repair.

\section{METHODS}

\section{Subjects}

The 16 patients were selected randomly from a PAWD surgical repair waiting list using the inclusion criteria that all subjects were male, less than 40 years old, "cardiovascularly fit", with no other groin pathology and no previous surgery. All subjects gave written consent. Three bilateral subjects and 13 unilateral subjects were recruited. "Cardiovascularly fit" was defined as no known heart problems and a moderate exercise tolerance, which was screened for in the questionnaire completed before surgery. Subjects over 40 years of age, women, or those with previous or current abdominal, lumbar spine, groin, knee, or hip pathology were excluded. The subjects with PAWD had been diagnosed and were under the care of a consultant general surgeon $(\mathrm{AB})$. Local research ethical committee approval was obtained.

Fifteen "normal" subjects matched for age, sex, sport played, and level of competition were assessed as a control group. These provided normal data for bilateral subjects and were used to compare for pain inhibition on the unaffected side with unilateral subjects.

\section{Assessment procedure}

All patients and their general practitioners were given an information sheet; after they had given written informed consent, they were assessed by the principle investigator $(\mathrm{AH})$. The assessment included; a questionnaire to establish pain levels, dominant side, functional ability, and the mechanism of injury. An isokinetic and isometric strength measurement on a Cybex NORM was performed. The isokinetic assessment was

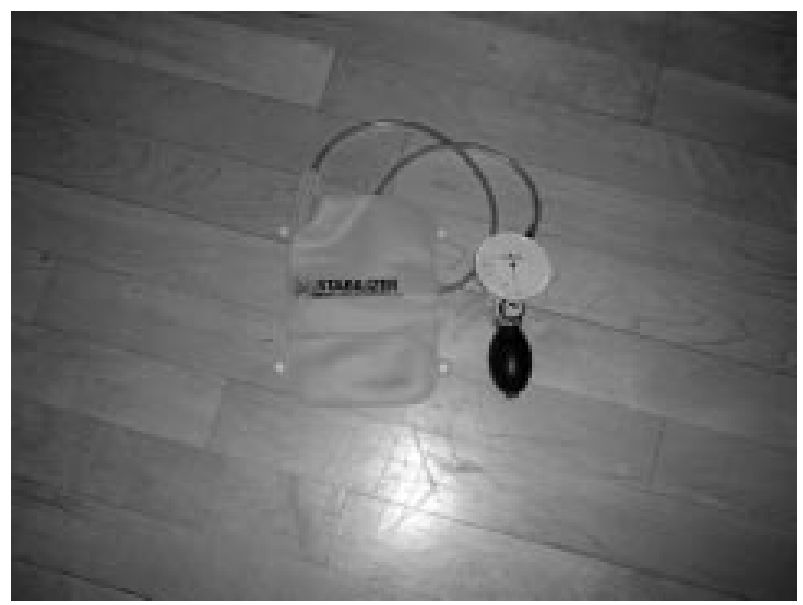

Figure 1 The pressure biofeedback unit.

of the concentric/concentric strength of the quadriceps and hamstrings at two speeds (60 and 120 degrees/s). The isometric test was a three repetition 15 second static holding test for the hip flexors, abductors, and adductors during standing. The standing position was used because it is more functional than lying, although this does compromise stability. With unilateral subjects the contralateral isokinetic measurements were used as control data. Verbal encouragement was standardised for all subjects to increase the reliability of the results, and all subjects were unable to see the monitor. Peak torque data were collected and expressed as a percentage of body weight to allow intersubject comparison.

A pressure biofeedback assessment of the stabilising abdominals was performed to assess deficits in transverse abdominals and oblique function and recruitment. The pressure biofeedback unit is an inflated cuff used to monitor the patient's ability to recruit and hold the local and global pelvic stability muscles (fig 1 ). This was done using the standardised pressure biofeedback measurement procedure. ${ }^{24}$ The exercises used were a controlled pelvic tilt (drawing in manoeuvre) in crook lying and a bent knee fallout exercise. The bent knee fallout procedure involved a controlled drawing in manoeuvre while controlling the bent leg into abduction and external rotation. Up to five practice repetitions were allowed to gain an optimum manoeuvre.

A reliability study for the pressure biofeedback was carried out using 10 normal subjects performing the bent knee fallout test three times.

The normal subjects were assessed for isokinetic muscle strength of the quadriceps and hamstrings and isometric strength of the hip flexors, abductors, and adductors. They also performed the pressure biofeedback tests for the transverse abdominals and obliques.

\section{Surgery}

The surgical repair was an open procedure involving a mesh repair. This was undertaken by a single surgeon $(\mathrm{AB})$ who specialises in groin repair surgery.

\section{Rehabilitation programme}

The subjects completed a six week exercise programme, which involved both attending physiotherapy once a week and a standardised home exercise programme. The rehabilitation programme is summarised below:

week 1: isometric abdominals and hip exercises, walk increased by $5 \mathrm{~min} /$ day, stairs;

week 2: active exercises for hips, transverse and oblique abdominals, exercise bike;

week 3: flexibility work, theraband hip exercises, transverse and oblique abdominals, jogging, swimming; 


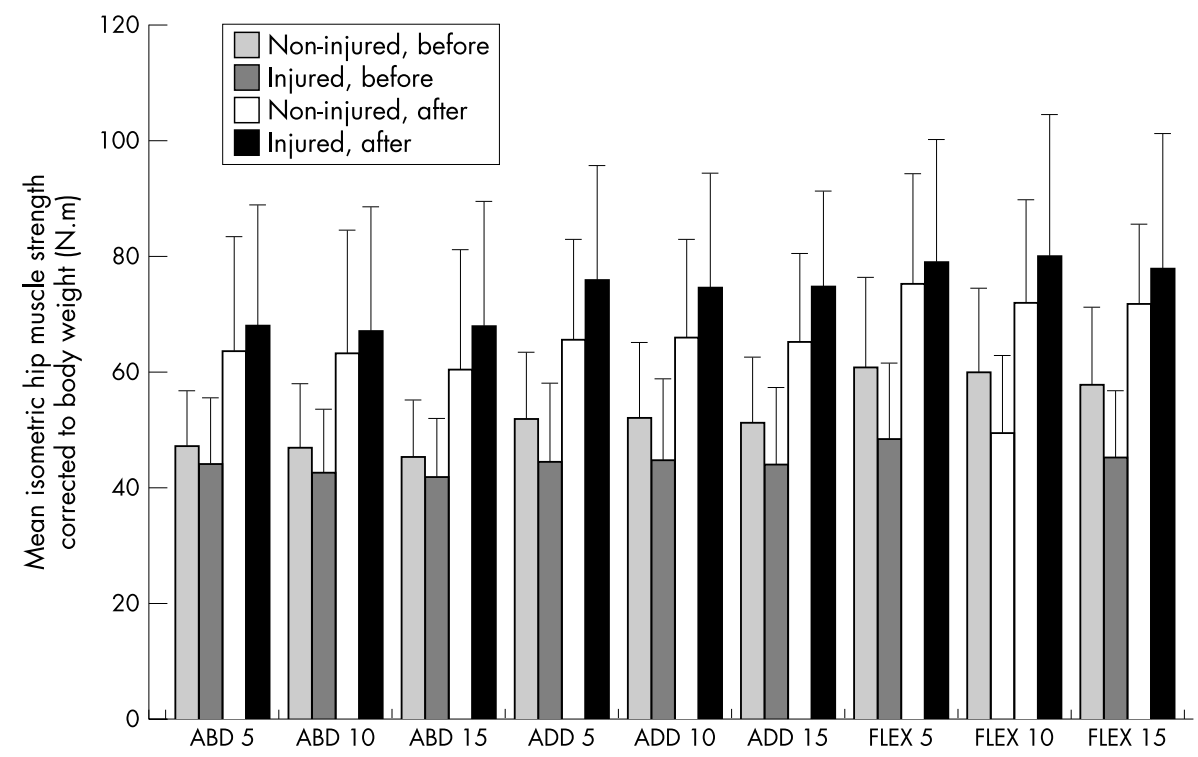

Figure 2 Comparison of mean hip muscle strength between the injured and non-injured limbs before and after surgery at 5, 10, and 15 seconds contraction. ABD, Abductor; ADD, adductor; FLEX, flexor.

week 4: running forward, progressing to abdominal work, low upper body weights;

week 5: sprinting, running in all directions, light ball skills, kicking, abdominals, and gradual return to training;

week 6: full training, matchplay and return to play.

\section{Assessment after surgery}

At six weeks after surgery, the patients completed another questionnaire, which established pain and functional levels. These could then be compared with data obtained before the operation. The pressure biofeedback, isokinetic, and isometric evaluation were performed again.

\section{Statistical analysis}

The dependent variables were muscle strength, pain level, and function. The independent variables were time with two levels and isokinetic speed with two levels. The reliability of the study may have been affected by tolerance to pain, pain relieving medication, adherence to the programme, familiarisation, and other exercise performed by the patient not included in the programme. As many of these variables were controlled for as far as possible by questioning the patient before the operation about treatment, exercise level, and activities.
Data were analysed using a computerised statistical package (SSPS for the PC). The repeated measures design and independent groups allowed two tailed $t$ tests assuming equal variances to be used to assess the difference between the two groups and within the injured group over time. The data were displayed graphically where appropriate. Parametric data obtained before and after surgery allowed a two way analysis of variance to be performed. An $\alpha$ level of 0.05 was selected a priori.

\section{RESULTS}

\section{Isokinetic data obtained before the operation}

At 60 and 120 degrees/s, the total work and peak torques for the quadriceps and hamstrings showed no significant difference bilaterally for the controls, the bilateral subjects, and the unilateral subjects. There was also no significant difference between the mean of the controls against the mean of the non-injured side or the mean of the injured side.

Assessment of isometric hip abductor, adductor, and flexor strength showed no significant difference between the injured and non-injured side of the bilateral subjects, controls, and the unilateral subjects. As there was no significant difference between or within groups, all injured sides were analysed

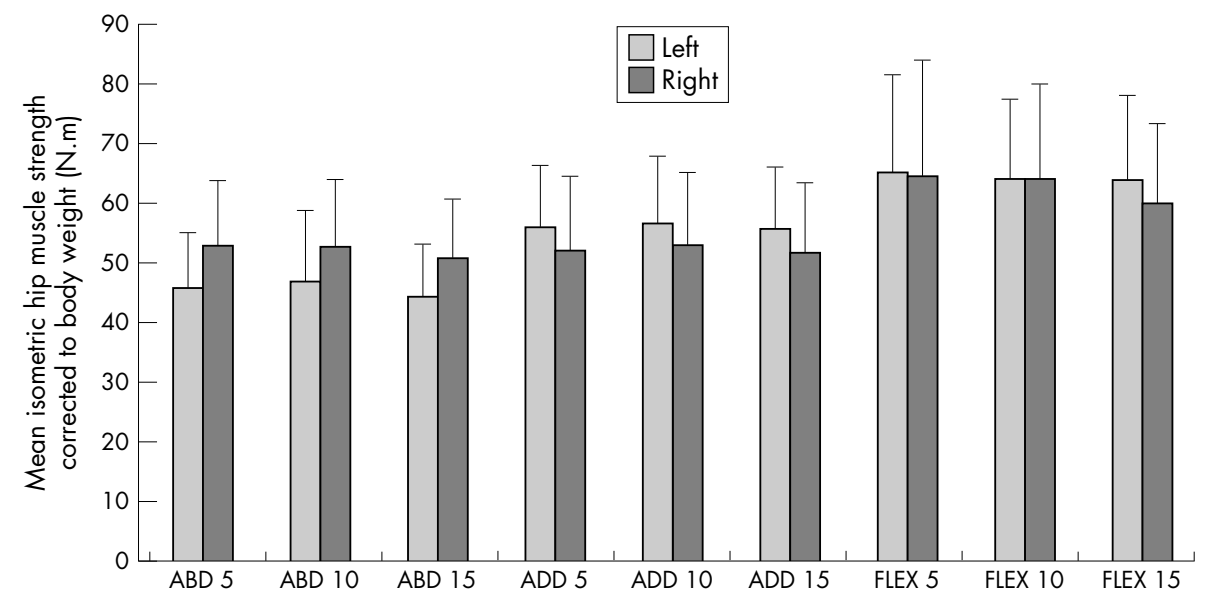

Figure 3 A bilateral comparison of the isokinetic hip muscle strength of the control group at 5, 10, and 15 seconds contraction. ABD, Abductor; ADD, adductor; FLEX, flexor. 
Table 1 Pass/fail rates for the transverse abdominals and the obliques using the pressure biofeedback

\begin{tabular}{lll}
\hline & $\begin{array}{l}\text { Transverse } \\
\text { abdominals }\end{array}$ & Obliques \\
\hline Pass before surgery & $87.5 \%$ & $13 \%$ \\
Fail before surgery & $12.5 \%$ & $87 \%$ \\
Pass after surgery & $100 \%$ & $96 \%$ \\
Fail after surgery & $0 \%$ & $4 \%$ \\
Control pass & $100 \%$ & $80 \%$ \\
Control fail & $0 \%$ & $20 \%$ \\
\hline
\end{tabular}

together against all non-injured sides. There was a significant deficit when either the unilateral injured or non-injured side abductors were compared with the right side of the control subjects.

Figure 2 shows that there was a general deficit in muscle strength on the affected side compared with the non-injured side before surgery, although this deficit was only significant at the 0.05 level for the hip flexors.

\section{Isokinetic data for control group}

There was no bilateral significant difference in hip strength in the control group (fig 3). The flexors were slightly stronger than the adductors and abductors.

\section{Pressure biofeedback data obtained before surgery}

Before surgery, $12.5 \%$ of the patients were unable to perform the transverse abdominals pressure biofeedback test, whereas all the control subjects could perform the test (table 1). The bent knee fallout test for the obliques was failed by $87 \%$ of the subjects; $80 \%$ of these were affected on the same extensor oblique as the pathological side. Only $20 \%$ of the control subjects failed this test.

\section{Subjective results before surgery}

Three quarters of subjects were injured on the dominant side. The most limited activity before surgical repair was sprinting, and the patients had more pain after exercise than during or before (fig 4).
Isokinetic data after surgery and rehabilitation

Analysis of peak torque and total work for the quadriceps and hamstrings showed no significant difference at the 0.05 level when results obtained before and after the operation were compared and when postoperative data on the injured and non-injured limb were compared.

The hip flexors, adductors, and abductors had all improved significantly in strength on the injured and non-injured sides after surgery and rehabilitation, whereas there was no significant difference from the non-injured side at this stage (fig 2).

\section{Pressure biofeedback data after surgery and rehabilitation}

After surgery and rehabilitation, 100\% of the subjects were able to perform the transverse abdominals test correctly, and $96 \%$ were able to perform the obliques test.

\section{Subjective results after surgery and rehabilitation}

Sprinting was still considered the most limited activity, although this was less limited than before. All activities and pain levels had improved (fig 4).

\section{DISCUSSION}

The complex nature of groin pain can make accurate diagnosis difficult. It has been shown that multiple pathologies are often involved and therefore a narrow approach to treatment often fails. ${ }^{84} \mathrm{~A}$ global approach to diagnosis and treatment is needed to find a cause and develop strategies for prevention and conservative treatment.

As there was no significant difference between the strength of the quadriceps and hamstrings bilaterally (before and after surgery), it can be assumed that the strength of these muscles was not affected by the condition and therefore strengthening of these muscles is questioned in terms of the relevance to the rehabilitation programme.

The finding of a general strength deficit in hip musculature on the affected limb may be attributed to disuse atrophy, as all athletes had reduced their activity levels since injury. Atrophy is not necessary indicative of pathology and could be attributed to apprehension; this would therefore improve with

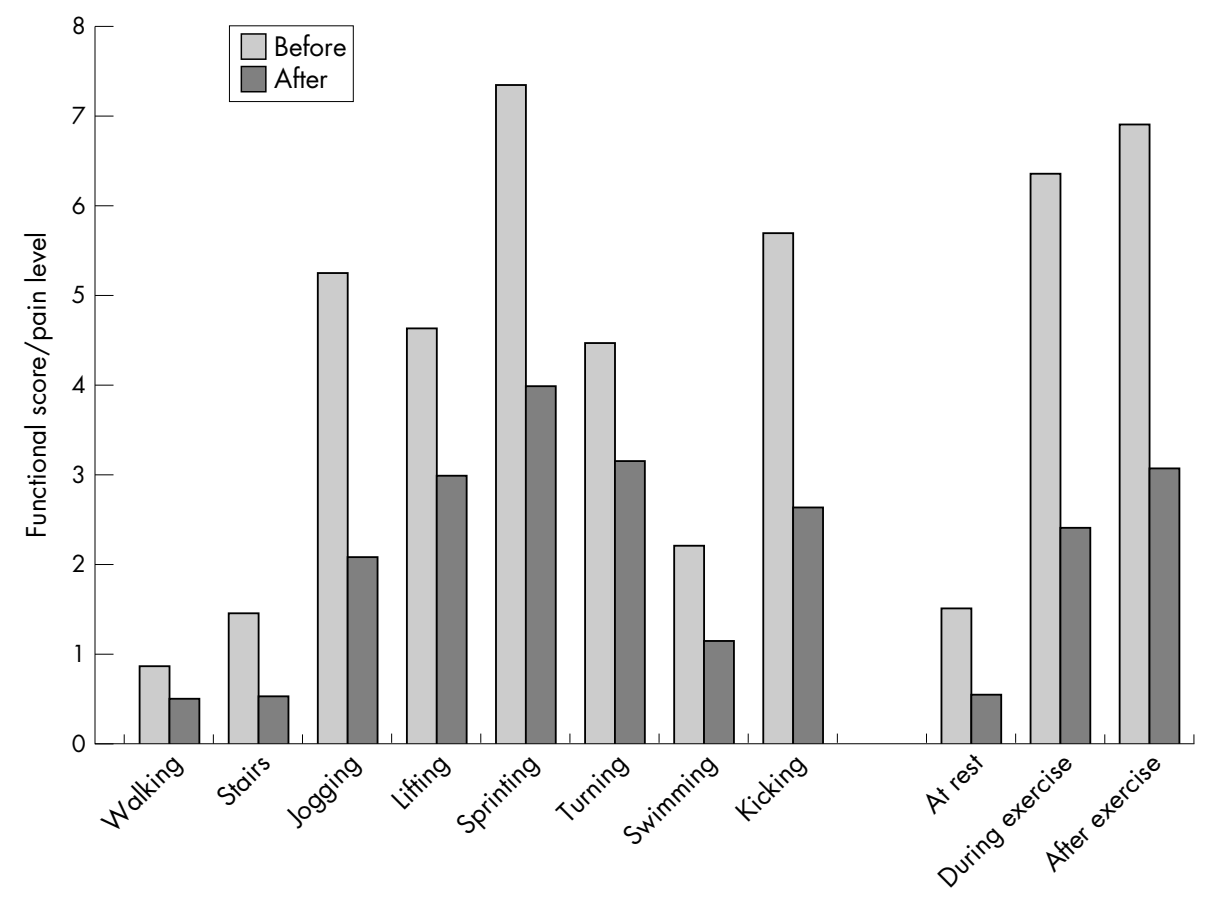

Figure 4 Mean function scores and pain levels before and after the operation. 
increasing confidence gained after the operation. No significant deficits in adductor and abductor muscle strength were found in this study, which may help to disprove theories that their role in pelvic stability, especially during kicking and turning, is an important contributing factor in this condition. Gibbon $^{25}$ described the pre-hernia complex that predisposes the athlete to PAWD and stated that the adductors have an important stabilising role when working with reversed attachments. Another theory stated that PAWD is caused by overly strong and tight hip flexors. ${ }^{10}$ This study found that the hip flexors were significantly weaker on the affected side. This weakness may have been attributed to pain inhibition, which would obviously have not been present before injury.

Only $12.5 \%$ of patients, compared with $100 \%$ of the controls, were unable to perform the transverse abdominal test before surgical repair. The obliques were affected in $87 \%$ of subjects compared with $20 \%$ of controls, often on the affected side. This higher incidence was possibly due to the obliques acting as global stabilisers of the pelvis, especially during rotation, and the direct injury to this muscle, whereas, because the transverse abdominals are local stabilisers of the lumbar spine and have less effect on pelvic stability, they are less likely to be affected in this condition. The current emphasis in rehabilitation programmes for this condition is to improve lower limb strength. The inability to control rotation is the most important finding of this study, suggesting that rehabilitation should be directed towards improving rotational control. Return to sport should occur when this is achieved. As the subjects have poor rotational control, one would expect that actions such as turning and kicking would be most limited; however, it appears that sprinting - a linear activity - is most affected, but this can be regarded as involving a contralateral rotation of the trunk to maintain trunk alignment and balance.

From these findings, the first null hypothesis that there was no strength deficit in the lower limb and abdominals in patients compared with matched controls was rejected.

The isokinetic findings that the hip musculature had improved in strength bilaterally after surgery and rehabilitation may be attributed to disuse atrophy and pain inhibition before surgery. The lack of difference between the injured and non-injured sides either before or after surgery leads us to question the theory that a unilateral strength deficit causes this injury.

At this stage, the $83 \%$ improvement in oblique abdominal strength after rehabilitation cannot be attributed to either the surgery or the rehabilitation process. Further research would be needed with a parallel study involving the six weeks rehabilitation programme alone, to assess the total effects of rehabilitation alone.

The patients reported that their pain levels had decreased and function had improved after surgery and rehabilitation. This supports previous evidence for the high levels of success of surgery.

Null hypothesis number 2 can be rejected, as deficits in strength, pain, and function were all improved after surgery. Therefore, surgery and the rehabilitation programme were considered successful.

Dugailly $e t a^{26}$ stressed the importance of evaluating hip muscle performance, as this may be an indicator of pelvic instability associated with pathology. The results from the isokinetic and biofeedback assessment may contribute to a screening programme for sports performers.

\section{Conclusions}

There was no significant difference in lower limb strength between injured and non-injured sides before surgery. Both limbs had increased in strength after surgery and rehabilitation. The oblique abdominals showed the greatest deficit before surgery and the greatest improvement after.

This study re-examines the causes of this condition and whether an appropriate conservative management programme would preclude the need for surgery.

\section{Take home message}

Rotational control of the pelvis would appear to be the most important factor in the prevention and rehabilitation of posterior abdominal wall deficiency.

Further research could include measuring the strength of the hip rotators and extensors. The range of rotation at the hip may also be an important factor in pelvic rotational stability. As the greatest deficit was in the oblique abdominals, a more sensitive test for the abdominals could be used alongside investigation of the electromyograph firing patterns of these muscles.

\section{ACKNOWLEDGEMENTS}

This study was completed in part for an MSc in Sports Injury and Therapy at Manchester Metropolitan University (AH). It was supported by grants from the Professional Footballers Association and The Chartered Society of Physiotherapy. We thank CHG Hospitals for the use of their equipment.

\section{Authors' affiliations}

A Hemingway, Cheshire Physiotherapy Centre, Altrincham, Cheshire, UK

L Herrington, University of Salford, Salford, UK

A L Blower, Wrightington, Wigan \& Leigh NHS Trust

\section{REFERENCES}

1 Ekstrand J, Hilding J. The incidence and differential diagnosis of acute groin injuries in male soccer players. Scand J Med Sci Sports 1999;9: 98-103.

2 Gilmore OJA. Gilmore's groin. Physiotherapy in Sport. 1995;18:14-15.

3 Norris C. Sports injuries diagnosis and management. 2nd ed. Oxford: Butterworth and Heinemann, 1998

4 Fricker PA. Management of groin pain in athletes. Br J Sports Med 1997;31:97-101.

5 Kemp S, Batt ME. The sports hernia a common cause of groin pain Physician and Sports Medicine 1998;26:36-44.

6 Lees A, Nolan L. The biomechanics of soccer a review. J Sports Sci 1998; 16:211-34.

7 Barfield WR. The biomechanics of kicking in soccer. Clin Sports Med 1998;17:711-28.

8 Evans DS. Sports hernia: the diagnosis and laproscopic management. Sports Exercise and Injury 1998;4:28-31

9 Polglase AL, Frydman GM, Farmer KC. Inguinal surgery for debilitating chronic groin pain in athletes. Med J Aust 1991;155:674-7.

10 Gilmore OJA. Groin pain in the soccer athlete: fact, fiction and treatment. Clin Sports Med 1998;17:787-93.

11 Janda V, Schmid HJA. Muscles as a pathogenic cause of back pain Proceedings of the International Federation of Orthopaedic Manipulative Therapists. New Zealand: Fourth Conference, 1980:17-18.

12 Hackney RG. The sportsman's hernia: a cause of chronic groin pain. $\mathrm{Br}$ J Sports Med 1993;27:58-62.

13 Simonet WT, Saylor HL, Sim L. Abdominal wall muscle tears in hockey players. Int J Sports Med 1995;16:126-8.

14 Lee D. The pelvic girdle. 2nd ed. London: Churchill Livingstone, 1999.

15 Sahrmann SA. Movement as a cause of musculoskeletal pain. Integrating Approaches, Proceedings of the Eighth Biennial Conference of the Manipulative Physiotherapists Association of Australia. Sydney: Sahrmann, 1993

16 Scott P. External herniae. An aid to clinical surgery. 3rd ed. Edinburgh: Churchill Livinstone, 1984:231.

17 Skandalakis JE, Gray SW, Skandalakis L. Surgical anatomy of the inguinal area. World J Surg 1989;13:490-8.

18 Fevre D. Hernia management and rehabiliation. Sportex Medicine 2000;5:33-8.

19 Brannigan AE, Kerin M, McEntee GP. Gilmore's groin repair in athletes. J Orthop Sports Phys Ther 2000;30:329-32

20 Williams P, Foster ME. Gilmore's groin or is it? Br J Sports Med 1995;29:206-8.

21 Orchard JW, Polglase AL, Frydman GM, et al. Inguinal surgery for debilitating chronic groin pain in athletes. Med J Aust 1998;155:674-7.

22 Hodges PW, Richardson CA. Inefficient muscular stabilization of the lumbar spine associated with low back pain. A motor control evaluation of the transverse abdominals. Spine 1996;21:2640-50.

23 Mulhearn S, George K. Abdominal muscle endurance and its association with posture and low back pain. Physiotherapy 1999:85:210-16.

24 Ekberg $\mathrm{O}$, Persson $\mathrm{NH}$, Abrahamsson $\mathrm{P}$, et al. Longstanding groin pain in athletes a multidisciplinary approach. Sports Med 1988;6:56-61.

25 Gibbon W. Diagnosis of groin pain. Royal College of Surgeons Sports Injury Conference. Lecture Notes. Lilleshall, UK: 2000.

26 Dugailly PM, Brassinne E, Mouraux D, et al. Isokinetic hip muscles performance in normal subjects. Isokinetics and Exercise Science 2000;8:42. 